\title{
Role of Nogo-A in the regulation of hepatocellular carcinoma SMMC-7721 cell apoptosis
}

\author{
CHUN-QIU HAO ${ }^{1,2}$, YUN ZHOU ${ }^{2}$, JIU-PING WANG ${ }^{2}$, MEI-JUN PENG ${ }^{2}$, YU-MEI XIE ${ }^{2}$, WEN-ZHEN KANG ${ }^{2}$, \\ LI SUN ${ }^{2}$, PING-ZHONG WANG ${ }^{2}$, CHUN-LING WAN $^{1}$, LIN HE $^{1}$, LEI CAI $^{1}$ and ZHANG-SHENG JIA ${ }^{2}$ \\ ${ }^{1}$ Bio-X Institutes, Key Laboratory for the Genetics of Developmental and Neuropsychiatric Disorders (Ministry of Education), \\ Shanghai Jiaotong University, Shanghai 200030; ${ }^{2}$ Centre of Liver Diseases, Fourth Military Medical University, \\ Tangdu Hospital, Xi'an, Shanxi 710038, P.R. China
}

Received June 29, 2013; Accepted February 18, 2014

DOI: $10.3892 / \mathrm{mmr} .2014 .2050$

\begin{abstract}
Nogo-A has been identified as an inhibitor of neurite outgrowth specific to the central nervous system. However, little is known about the role of Nogo-A in hepatocellular carcinoma (HCC), the most common primary malignant tumor with a high mortality rate. This study aimed to investigate the role of endogenous Nogo-A in human liver cancer cells. Reverse transcription polymerase chain reaction was used to detect the expression of Nogo-A in four liver cancer cell lines. A lentivirus vector was then constructed to mediate RNA interference (RNAi) targeting of Nogo-A (LV-Nogo-A-siRNA) and was confirmed to successfully suppress the expression of the Nogo-A gene in SMMC-7721 cells. Furthermore, Nogo-A was observed to be highly expressed in liver cancer cell lines. RNAi of Nogo-A using the LV-Nogo-A-siRNA construct significantly decreased Nogo-A protein expression and specifically inhibited the growth of SMMC-7721 cells. This growth inhibitory effect may be attributed to an increase in $\mathrm{G} 2 / \mathrm{M}$ phase arrest and apoptosis in SMMC-7721 cells containing Nogo-A-siRNA. The results of this study demonstrate that Nogo-A may represent a novel therapeutic target for the treatment of liver cancer, in addition to its potent roles in neural systems.
\end{abstract}

Correspondence to: Dr Zhan-Sheng Jia, Centre of Liver Diseases, Fourth Military Medical University, Tangdu Hospital, 1 Xinsi Road, Xi'an, Shangxi 710038, P.R. China

E-mail: haochq@yahoo.com.cn

Dr Lei Cai, Bio-X Institutes, Key Laboratory for the Genetics of Developmental and Neuropsychiatric Disorders (Ministry of Education), Shanghai Jiaotong University, 55 Guangyuanxi Road, Shanghai 200030, P.R. China

E-mail: cailei2010@126.com

Key words: Nogo-A, myelin, apoptosis, lentivirus

\section{Introduction}

Nogo-A, a myelin-associated endoplasmic reticulum protein, is encoded by the reticulon-4 (RTN4) gene located at chromosome 2p13-14 (1). The human RTN4 gene encodes three alternatively spliced variants, Nogo-A, Nogo-B and Nogo-C. Nogo-A is the largest Nogo isoform, which exhibits a unique $\mathrm{N}$-terminal extension and is mainly expressed by oligodendrocytes in the brain. By contrast, Nogo-B demonstrates a ubiquitous pattern of expression and Nogo-C is highly expressed in skeletal muscles $(2,3)$. The three Nogo isoforms share a common C-terminal domain of 188 amino acids, which contains two putative transmembrane domains and an endoplasmic reticulum retention motif (4). In vitro studies have reported that Nogo-A contributes to the axonal growth inhibitory function of central myelin; however, there have been contradictory results regarding this growth inhibitory effect in Nogo-A knockout mice $(5,6)$. Whilst the role of Nogo-A in the nervous system is well established, including activation of growth cone collapse, axonal outgrowth inhibition and synaptic plasticity (7-9), the role of endogenous Nogo-A in non-neural systems is yet to be elucidated.

Hepatocellular carcinoma (HCC) is the most common primary malignant tumor and accounts for $\sim 5.6 \%$ of all tumors (10). As a result of the poor prognosis associated with $\mathrm{HCC}$, a high mortality rate is observed among patients with the tumor. Therefore, exploring the molecular mechanisms associated with HCC and developing more effective targeted therapies are of utmost importance. The Arg114Gly mutation in the Nogo-C gene, which affects the dimensional structure of the Nogo-66 domain, was observed to promote apoptosis in a liver cancer cell line (11). The Nogo-66 domain has been identified as a conserved functional group among the three Nogo isoforms; therefore, it is hypothesized that Nogo-A may have a role in HCC.

The present study aimed to explore the role of the neural-associated Nogo-A gene in liver cancer cells. Nogo-A was observed to exhibit high expression in the HCC SMMC-7721 cell line. Furthermore, a novel lentivirus vector that mediated RNA interference (RNAi) targeting of Nogo-A (LV-Nogo-A-siRNA) was employed to study the effect of Nogo-A knockdown on SMMC-7721 cell growth in vitro. 


\section{Materials and methods}

Cell culture. The human liver cancer cell lines HepG2, Huh-7, BEL-7402 and SMMC-7721 (Shanghai GeneChem Co., Shanghai, China) were maintained in Dulbecco's modified Eagle medium (DMEM; Gibco-BRL, Grand Island, NY, USA) supplemented with $10 \%(\mathrm{v} / \mathrm{v})$ heat-inactivated fetal bovine serum (FBS; Gibco-BRL) at $37^{\circ} \mathrm{C}$ with $5 \% \mathrm{CO}_{2}$.

Construction of lentiviral vectors, and preparation and transduction of the lentivirus. The complementary DNA sequence of Nogo-A was designed from full-length Nogo-A by Shanghai GeneChem Co., Ltd. The sequence of the small interfering RNA (siRNA) targeting Nogo-A was as follows: ccggGC TATATCTGAGGAGTTGGTTTTCAAGAGAACCAACTC CTCAGATATAGCTTTTTgaatt. Following the testing of knockdown efficiencies, stem-loop oligonucleotides were synthesized and cloned into the lentivirus-based vector, pGCSIL-enhanced green fluorescence protein (eGFP). A non-targeting stem-loop DNA was generated as a negative control. An improved lentivirus system of pGC-LV was developed by Shanghai GeneChem Co, Ltd. This system consisted of the following plasmids: pHELPER1.0, a packaging plasmid in which the accessory genes, vif, vpr, vpu and nef, and a regulatory gene, tat, were deleted; pHELPER2.0, an envelope plasmid for vesicular stomatitis virus G glycoprotein (VSV-G); and pGCSIL-eGFP, a transferring plasmid with a multiple cloning site and the gene encoding eGFP (Fig. 1A).

The recombinant Nogo-A-siRNA lentivirus vector was generated using co-transfection of 293T cells with $20 \mu \mathrm{g}$ pGCSIL-Nogo-A-siRNA-eGFP, $15 \mu \mathrm{g}$ pHELPER1.0, $10 \mu \mathrm{g}$ pHELPER2.0 and Opti-MEM ${ }^{\circledR}$ (Invitrogen Life Technologies, Carlsbad, CA, USA) of the same volume in $15-\mathrm{cm}$ dishes with Lipofectamine ${ }^{\circledR} 2000$ (Invitrogen Life Technologies). The $293 \mathrm{~T}$ cells were cultured in DMEM containing 10\% FBS. Culture supernatants were collected after two days, filtered through a $0.45-\mu \mathrm{m}$ pore size filter and concentrated using Centricon ${ }^{\circledast}$ Plus-20 (Millipore Corporation, Hayward, CA, USA). The viral filtrate was collected and stored at $-80^{\circ} \mathrm{C}$.

Cells were incubated with the lentivirus in a small volume of serum-free DMEM at $37^{\circ} \mathrm{C}$ for $4 \mathrm{~h}$. Medium was then replaced with DMEM containing 10\% FBS, and cells were cultured further as indicated for the following experiments. eGFP revealed that the infection efficiency in SMMC-7721 cells was $\sim 90 \%$ and at a multiplicity of infection (MOI) of 30 . No viral toxicity was observed at this concentration in the SMMC-7721 cells; therefore, the following experiments were performed using viruses at such MOIs, unless indicated. The lentivirus package with eGFP was used for the cell proliferation assay, and that without eGFP was used for the cell apoptosis assay.

Western blot analysis of exogenous flag-Nogo-A expression. To assess the gene knockdown efficiency of Nogo-A-siRNA, an exogenous 3 flag-Nogo-A fusion protein was constructed into a GV143 vector (Shanghai GeneChem Co.). The constructed 3flag-Nogo-A vector was co-transfected with pGCSIL-Nogo-A-siRNA-eGFP or pGCSIL-scr-siRNA-eGFP, respectively, into $293 \mathrm{~T}$ cells. The transfection ratio was estimated using fluorescence microscopy. Total proteins were extracted $48 \mathrm{~h}$ following transfection and the protein was quantified using the Coomassie brilliant blue assay. Subsequently, $20 \mu \mathrm{g}$ protein was boiled in loading buffer, separated on $10 \%$ SDS-polyacrylamide gels, electro-transferred to polyvinylidene fluoride (PVDF) membranes and probed with mouse anti-flag (Sigma Aldrich, St. Louis, MO, USA) and mouse anti-GAPDH (Santa Cruz Biotechnology, Inc., Santa Cruz, CA, USA) antibodies overnight at dilutions of 1:3,000 and 1:5,000 respectively. Membranes were then incubated with a goat anti-mouse immunoglobulin $\mathrm{G}(\mathrm{IgG})$ peroxidase-conjugated secondary antibody (Santa-Cruz Biotechnology, Inc., 1:5,000), prior to development using the Amersham enhanced chemiluminescence (ECL) plus western blotting detection system (Amersham Pharmacia Biotech, Piscataway, NJ, USA).

RNA isolation and quantitative polymerase chain reaction (qPCR) analysis. Total RNA was extracted using TRIzol ${ }^{\circledR}$ reagent (Invitrogen Life Technologies) in accordance with the manufacturer's instructions. A total of $2 \mu \mathrm{g}$ RNA was subjected to reverse transcription. The PCR primer sequences were as follows: GAPDH, 5'-TGACTTCAACAGCGACAC CCA-3' (forward) and 5'-CACCCTGTTGCTGTAGCCAAA-3' (reverse); Nogo-A, 5'-AGGAGCAGCCAGGTAACAC-3' (forward) and 5'-GAGACAGAGAAGGAAGAGAAGC-3' (reverse), as described previously (11). PCR products were separated on a $1 \%$ agarose gel, and visualized and photographed under ultraviolet light for semi-quantitation.

Cell growth assay. The cell growth rate was determined using the Cellomics ${ }^{\circledast}$ ArrayScan ${ }^{\circledR}$ VTI machine (Thermo Fisher Scientific, San Jose, CA, USA). Cells were transduced with lentivirus vectors for $72 \mathrm{~h}$, prior to being seeded into flat-bottom 96-well plates at 2,000 cells per well. Cells were observed at one, two, three, four and five days using the Cellomics ArrayScan VTI machine. In the Cellomics ArrayScan VTI system (Thermo Fisher Scientific), an automated inverted epifluorescence microscope was used to record images from multiple fields in each individual well. Fluorescence images were acquired using a high-resolution charge-coupled device (CCD) camera. Cells were identified based on the presence of valid nuclei and cell body measurements, determined by analyzing size, shape and eGFP fluorescence intensity. Images were then analyzed using the Cellomics software and cells were counted (12). Cell growth curves were generated following three experimental repeats.

Colony formation assay. The Cellomics ArrayScan VTI system was used to perform colony formation assays to assess the anchorage-independent growth ability of cells as a characteristic of in vitro tumorigenicity. SMMC-7721 cells were infected with the virus for $24 \mathrm{~h}$, then detached using Trypsin (Chemreagent, Shanghai, China) and plated in 96-well plates at 500 cells/well. The number of foci $(>100 \mu \mathrm{m})$ was counted after 14 days. Each experiment was performed in triplicate.

Flow cytometric analysis. Flow cytometric analysis was performed to determine the distribution of cells throughout the cell cycle and those undergoing apoptosis, and was performed as described previously (13). SMMC-7721 cells were seeded and transduced with lentivirus vectors and then cultured for 
$96 \mathrm{~h}$ in complete medium. Following one wash with Hanks balanced salt solution (Sigma Aldrich) adherent cells were detached using trypsin, and cells were washed once with ice cooled phosphate-buffered saline (PBS). Prior to analysis, the transduction efficiency and cell death rate were estimated using a fluorescence microscope. Cells with $>90 \%$ transduction efficiency and $<1 \%$ death rate were used for the subsequent fluorescence-activated cell sorting (FACS) analysis.

For analysis of apoptosis, cells were washed with $1 \mathrm{X}$ binding buffer before $1 \times 10^{6}-1 \times 10^{7}$ cells were pelleted and resuspended in $1 \mathrm{ml} 1 \mathrm{X}$ staining buffer. A total of $100 \mu \mathrm{l}$ of the cell suspension was added to $5 \mu \mathrm{l}$ Annexin V-allophycocyanin (APC) and incubated in the dark at room temperature for 10-15 min. Data from $\geq 10,000$ cells were collected and analyzed using CellQuest software (Becton Dickinson, San Diego, CA, USA). The apoptosis ratio was calculated as the number of apoptotic cells/total number of cells.

For cell cycle analysis, cells were fixed using $0.5 \mathrm{ml} 70 \%$ iced alcohol and incubated at $4^{\circ} \mathrm{C}$ for $1 \mathrm{~h}$, prior to being washed once with ice cooled PBS. The cell pellet was resuspended with staining buffer at $4^{\circ} \mathrm{C}$ for $30 \mathrm{~min}$ [40X $2 \mathrm{mg} / \mathrm{ml}$ propidium iodide (PI): $100 X 10 \mathrm{mg} / \mathrm{ml}$ RNase: $1 X$ PBS=25:10:1,000]. The suspension was filtered through a $50-\mu \mathrm{m}$ nylon mesh, and flow cytometry was used to analyze the DNA content of the stained nuclei with a BD FACSCalibur machine (Becton Dickinson). Cell cycle distribution was assessed using Multicycle cell cycle analysis software (Phoenix Flow Systems, San Diego, CA, USA).

Statistical analysis. Each experiment was repeated at least three times. Bands from western blot analysis or qPCR were quantified using Quantity One ${ }^{\circledR}$ software (Bio-Rad Laboratories Inc., Berkley, CA, USA). Relative protein or mRNA levels were calculated using GAPDH as a normalization control. T-test analysis was performed to determine the significance of the differences between means. All statistical analyses were performed using Excel 2003 software (Microsoft, Redmond, WA, USA). A value of $\mathrm{P}<0.05$ was considered to indicate a statistically significant difference.

\section{Results}

Expression of Nogo-A in liver cancer cell lines. To determine the expression of Nogo-A in different liver cancer cell lines, qPCR analysis was performed. As shown in Fig. 2, Nogo-A expression was observed in four different liver cancer cell lines.

Effect of RNAi targeting Nogo-A on Nogo-A expression using the LV-Nogo-A-siRNA lentivirus vector. To examine the interrelation between Nogo-A and liver cancer cells, a lentivirus-delivered Nogo-A-specific siRNA vector (LV-Nogo-A-siRNA) and a negative control scramble-siRNA vector (LV-scr-siRNA) were constructed. The two vectors were then respectively transduced into SMMC-7721 cells for three days. qPCR and western blot analyses demonstrated that the level of Nogo-A expression was lower in SMMC-7721/Nogo-A-siRNA cells than in SMMC-7721/scr-siRNA cells $(\mathrm{P}<0.001)$. Furthermore, Nogo-A-siRNA was also observed to knockdown expression of exogenous 3flag-Nogo-A (Fig. 3). These data show that the
A Transfer vector (pGCSIL-eGFP)

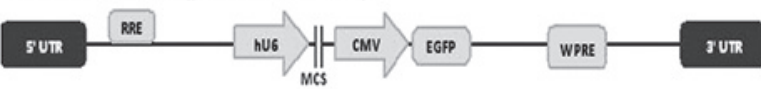

Packaging vector (pHELPER1.0)

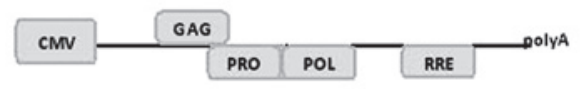

Env-coding vector (pHELPER2.0)
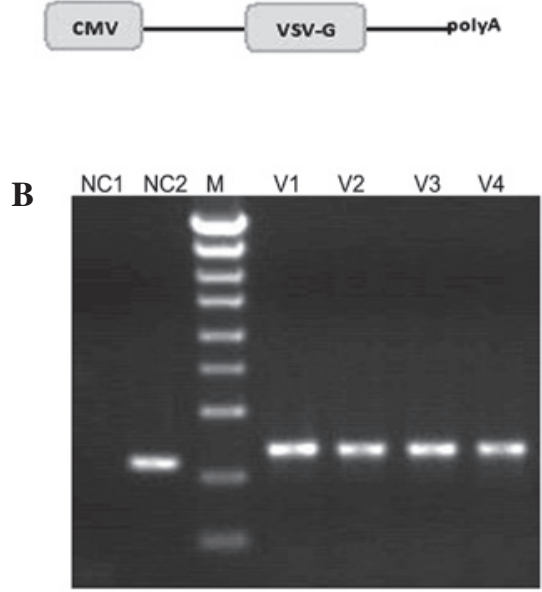

Figure 1. Packaging system of pGC-LV and identification of pGCSIL-eGFP using PCR. (A) Recombinant self-inactivating lentivirus vectors were produced by co-transfection of the envelope plasmid pHELPER2.0, packaging plasmid pHELPER1.0 and transfer vector plasmid. (B) Detection of the positive clone-containing pGCSIL-eGFP using PCR. Lane 1, negative control with $\mathrm{H}_{2} \mathrm{O}(\mathrm{NC} 1)$; lane 2, empty vector of $306 \mathrm{bp}$ (NC2); lane 3, DNA ladder (M) from top to bottom, $5 \mathrm{~kb}, 3 \mathrm{~kb}, 2 \mathrm{~kb}, 1.5 \mathrm{~kb}, 1 \mathrm{~kb}, 750 \mathrm{bp}, 500 \mathrm{bp}, 250 \mathrm{bp}$ and $100 \mathrm{bp}$; lanes 4-7, recombinant vector of $343 \mathrm{bp}$ (V1-V4). LTR, long terminal repeat; RRE, Rev response element; hU6, human U6 promoter; MCS, multiple cloning sites; CMV, cytomegalovirus promoter; eGFP, enhanced green fluorescent protein marker gene; WPRE, woodchuck post-transcriptional regulatory element; cPPT, central polypurine tract; Env, envelope protein; VSV-G, vesicular stomatitis virus G protein envelope; GAG, group-specific antigen; POL, reverse transcriptase; PRO, protease; PCR, polymerase chain reaction.

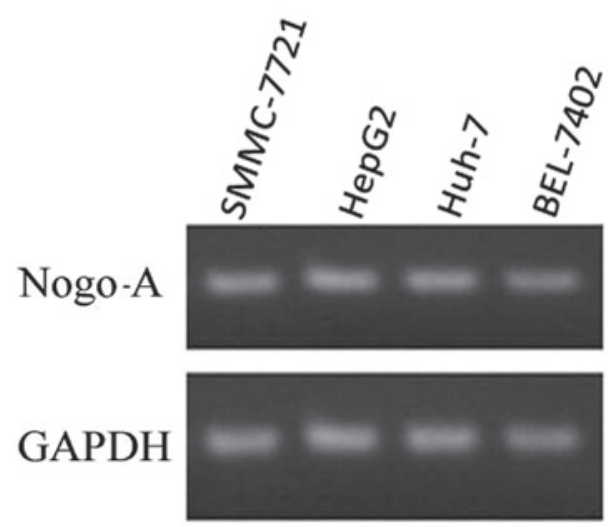

Figure 2: Detection of Nogo-A mRNA levels in four liver cancer cell lines using quantitative polymerase chain reaction.

constructed recombinant lentiviral vector of Nogo-A-siRNA is capable of efficiently downregulating endogenous and exogenous Nogo-A expression in SMMC-7721 cells.

Depletion of Nogo-A inhibits cell growth. The present study further investigated the effect of Nogo-A siRNA 

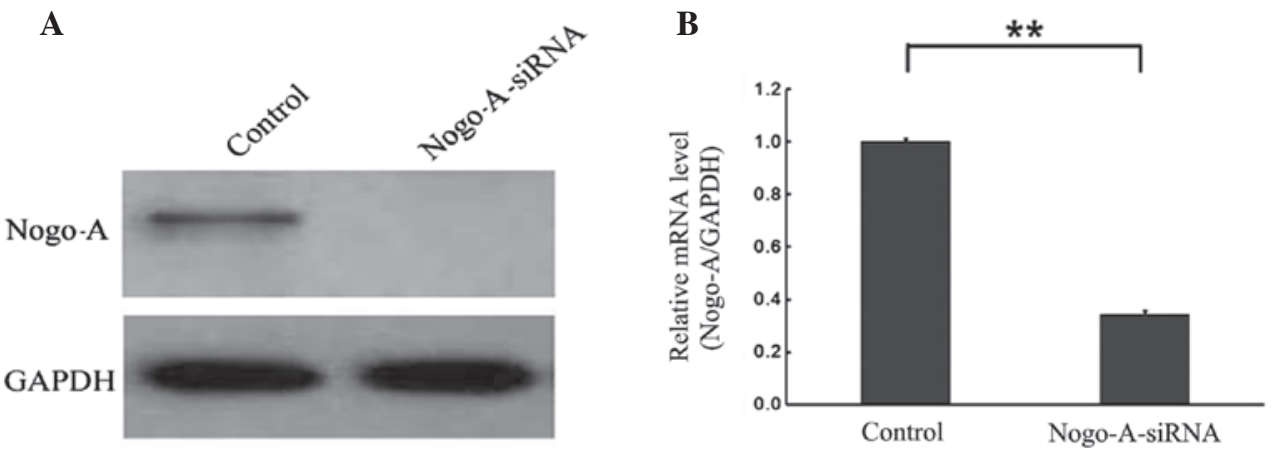

Figure 3. Constructed lentivirus vector of Nogo-A-siRNA and its efficiency in silencing Nogo-A expression in SMMC-7721 cells. (A) Western blot analysis of exogenous flag-Nogo-A protein expression in transduced $293 \mathrm{~T}$ cells containing Nogo-A-siRNA or scr-siRNA; (B) quantitative polymerase chain reaction of endogenous Nogo-A mRNA levels in SMMC-7721 cells transduced with LV-Nogo-A-siRNA or LV-scr-siRNA (control); ${ }^{* *} \mathrm{P}=9.03 \times 10^{-7}$. siRNA, short interfering RNA; scr, scramble.

A
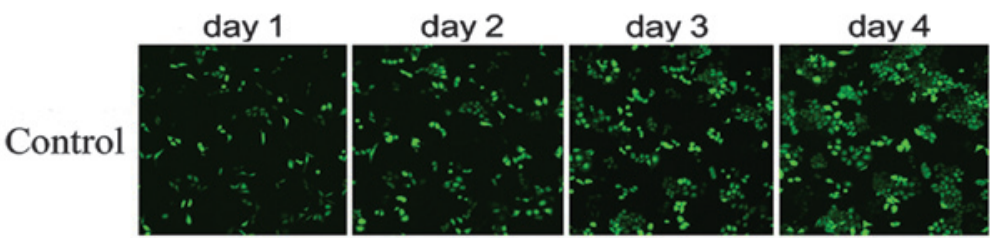

day 5
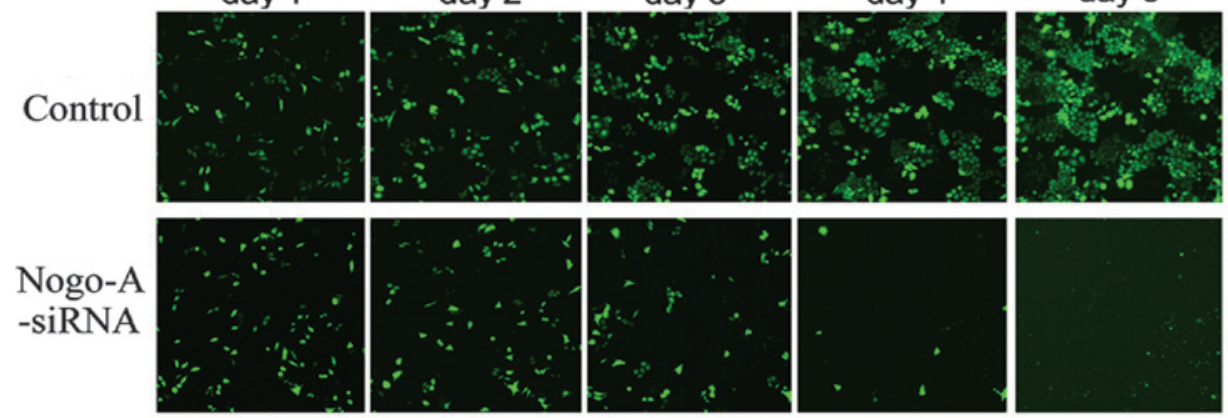

B

C
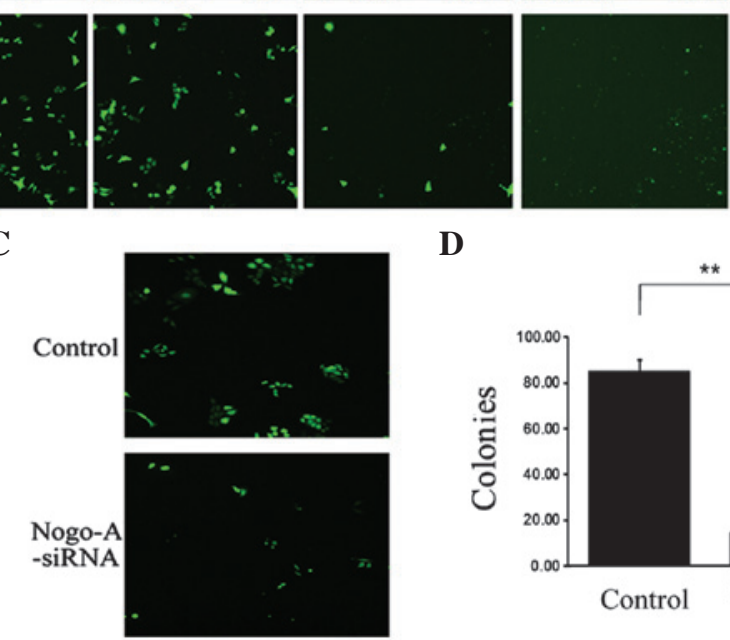

D
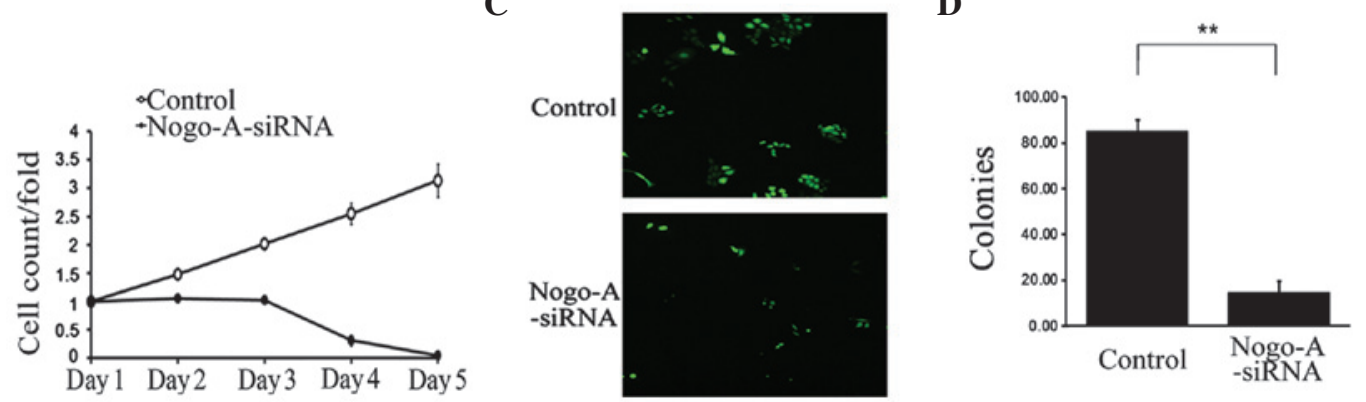

Figure 4. (A) Nogo-A depletion decreases SMMC-7721 cell proliferation and colony formation as determined using Cellomics ArrayScan (Thermo Fisher Scientific, San Jose, CA, USA). (B) LV-Nogo-A-siRNA depresses the SMMC-7721 cell growth curve compared with LV-scr-siRNA, in a time-dependent manner. As shown using a colony formation assay, LV-Nogo-A-siRNA decreases the (C) size and (D) number of SMMC-7721 cell colonies compared with LV-scr-siRNA; ${ }^{* *} \mathrm{P}=1.05 \times 10^{-6}$. siRNA, short interfering RNA.

treatment on SMMC-7721 cell viability. As shown in Fig. 4 , the growth of SMMC-7721 cells treated with Nogo-A siRNA was significantly inhibited compared with those treated with scr-siRNA (Fig. 4A and B). This growth inhibitory effect was also confirmed by colony formation assay, which demonstrated that LV-Nogo-A-siRNA transfection significantly decreased the number and size of SMMC-7721 cell colonies (Fig. 4C and D). These data indicate that knockdown of Nogo-A is capable of inhibiting proliferation in the HCC SMMC-7721 cell line. Furthermore, the effect of Nogo-A depletion was assessed in three other cancer cell lines: H1299, RKO and SKOV3. Compared with the scr-siRNA, Nogo-A siRNA was not observed to significantly inhibit growth in these cancer cell lines (data not shown). These results suggested that the growth inhibitory effect of Nogo-A siRNA may be liver cancer-specific.
Cell cycle arrest in G2/M phase by LV-Nogo-A siRNA in $S M M C-7721$ cells. Experiments were also performed to assess the role of the cell cycle in mediating the anti-proliferative effects of Nogo-A inhibition in SMMC-7721 cells. Cell cycle distribution was analyzed at $96 \mathrm{~h}$ following siRNA transduction, using flow cytometry. Cell cycle analysis revealed that inhibition of Nogo-A expression using siRNA caused a significant increase in the proportion of the cell population in $\mathrm{G} 2 / \mathrm{M}$ phase compared with that of the control population transduced with scr-siRNA (Fig. 5A; $\mathrm{P}=0.0014$ ). These data indicate that knockdown of Nogo-A expression is capable of inducing G2/M arrest in SMMC-7721 cells.

Induction of apoptosis by LV-Nogo-A siRNA in SMMC-7721 cells. To determine whether Nogo-A depletion is capable of inducing apoptosis in SMMC-7721 cells, flow 
A

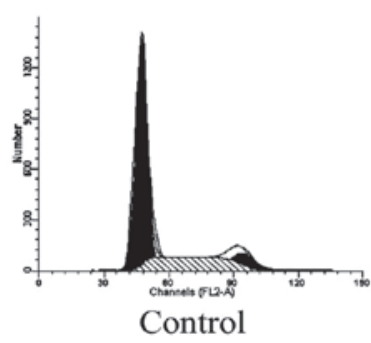

B

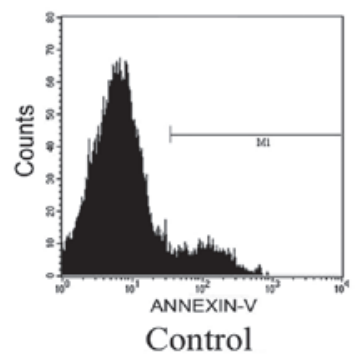

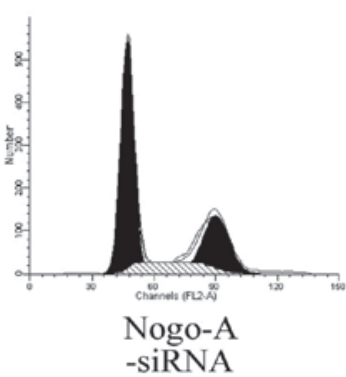

-siRNA

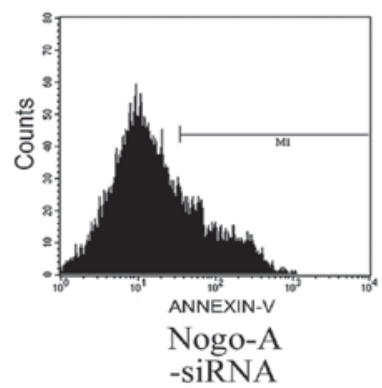

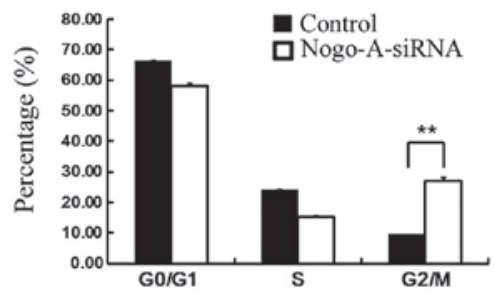

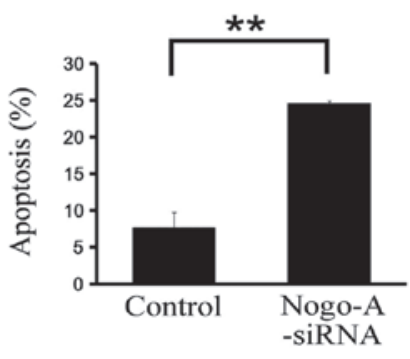

Figure 5. Effect of LV-Nogo-A-siRNA on cell cycle and apoptosis determined by flow cytometric analysis. (A) Knockdown of Nogo-A expression using LV-Nogo-A-siRNA induced G2/M phase arrest which disrupted cell cycle progression, compared with LV-scr-siRNA; P=0.0014. (B) Knockdown of Nogo-A expression using LV-Nogo-A-siRNA increased cell apoptosis, compared with LV-scr-siRNA; P=0.004. siRNA, short interfering RNA; scr, scamble.

cytometric analysis was performed $96 \mathrm{~h}$ following siRNA transduction (Fig. 5B). Apoptosis was observed in $24.7 \%$ of the SMMC-7721 cells transduced with Nogo-A-siRNA compared with $7.6 \%$ of the control cells transduced with scr-siRNA $(\mathrm{P}=0.004)$. Since the transduction efficiency was $>90 \%$, these data suggest that knockdown of Nogo-A expression promotes an increase in apoptosis in the HCC SMMC-7721 cell line.

\section{Discussion}

Liver cancer is associated with the highest mortality rate among all types of cancer. However, even when diagnosed early, few patients represent candidates for surgery, due the high likelihood of HCC cell relapse and metastasis following surgery. Therefore, the development of novel therapies for the treatment of liver cancer is of great importance. It has been previously reported that Nogo-A has a significant role in the development of myelin and other central nervous tissues $(4,9)$. Numerous studies have suggested that the Nogo-A, Nogo-B and Nogo-C isoforms have a role in apoptosis, particularly in cancer cells (14-16). This study has presented the Nogo-A expression profile in four liver cancer cell lines, and has investigated the inhibitory effect of endogenous Nogo-A on HCC SMMC-7721 cells using an improved lentivirus packaging system.

Lentivirus vectors, which integrate their complementary DNA (cDNA) into dividing and non-dividing cells, are capable of permanently integrating into their target cells (17). In comparison with the small hairpin RNA (shRNA) expression vector systems, whose transduction efficiency and transient shRNA expression are low (18), lentivirus-delivered siRNAs are capable of specific, highly stable and functional silencing of gene expression in a variety of human cells (19). This study employed an improved lentivirus vector with a deletion of the
U3 region, including the TATA box, of the 3 ' long terminal repeat (LTR), which deactivated the LTR promoter and significantly enhanced the safety level of transgene expression. Using this lentivirus system, a lentivirus vector that mediated RNAi targeting of Nogo-A (LV-Nogo-A-siRNA) was constructed, and was observed to effectively and specifically downregulate Nogo-A expression in SMMC-7721 cells by $\leq 90 \%$.

The proliferation of SMMC-7721 cells was observed to be markedly reduced following infection with LV-Nogo-A-siRNA for $72 \mathrm{~h}$. Furthermore, the growth inhibitory effect associated with LV-Nogo-A-siRNA infection was identified as being SMMC-7721 cell-specific. Moreover, the present study revealed that Nogo-A depletion may achieve such growth inhibition by arresting the SMMC-7721 cells in the G2/M phase of the cell cycle and consequently promoting apoptosis. These results are in accordance with the findings of Sutendra et al (20), who suggested that the absence of Nogo-B may increase the susceptibility of pulmonary arterial smooth muscle cells to apoptosis.

Contrary to the findings of the present study, Chen et al (14) observed that the overexpression of mutant Nogo-C was capable of inducing apoptosis in HCC SMMC-7721 cells. Among the three Nogo isoforms, Nogo-C is the shortest, while Nogo-A is the longest. These conflicting findings suggest that the exogenous mutant Nogo-C may be capable of antagonizing endogenous Nogo-A, thereby reducing the activity of Nogo-A and increasing apoptosis. Further evidence is required to verify this hypothesis.

In conclusion, the present study found that Nogo-A depletion was capable of inhibiting HCC SMMC-7721 cell proliferation by promoting $\mathrm{G} 2 / \mathrm{M}$ cell cycle arrest and apoptosis. To the best of our knowledge, this is the first investigation into the effect of endogenous Nogo-A in a liver cancer cell line. The present findings suggest that Nogo-A may represent an effective molecular target for the therapeutic treatment of liver cancer, in addition to its significant roles in neural systems. 


\section{Acknowledgements}

The authors would like to thank Professor Krzysztof Trzciński and Dr. Bing-Jun Qian for their generous assistance and editing skills. This study was supported by the 973 Program (no. 2012CB910100) to CLW, the National Nature Science Foundation of China (no. 31101015) and the Scientific Research Foundation for the Returned Overseas Chinese Scholars to LC, State Education Ministry (no. 12Z102050009).

\section{References}

1. Yang J, Yu L, Bi AD and Zhao SY: Assignment of the human reticulon 4 gene (RTN4) to chromosome 2 p14-->2p13 by radiation hybrid mapping. Cytogenet Cell Genet 88: 101-102, 2000.

2. GrandPré T, Nakamura F, Vartanian T and Strittmatter SM: Identification of the Nogo inhibitor of axon regeneration as a Reticulon protein. Nature 403: 439-444, 2000.

3. Woolf CJ: No Nogo: now where to go? Neuron 38: 153-156, 2003.

4. Chen MS, Huber AB, van der Haar ME, et al: Nogo-A is a myelin-associated neurite outgrowth inhibitor and an antigen for monoclonal antibody IN-1. Nature 403: 434-439, 2000.

5. Zheng B, Ho C, Li S, Keirstead H, Steward O and Tessier-Lavigne M: Lack of enhanced spinal regeneration in Nogo-deficient mice. Neuron 38: 213-224, 2003.

6. Kim JE, Li S, GrandPré T, Qiu D and Strittmatter SM: Axon regeneration in young adult mice lacking Nogo-A/B. Neuron 38: 187-199, 2003.

7. Fournier AE, GrandPré T and Strittmatter SM: Identification of a receptor mediating Nogo-66 inhibition of axonal regeneration. Nature 409: 341-346, 2001.

8. Xie $\mathrm{F}$ and Zheng B: White matter inhibitors in CNS axon regeneration failure. Exp Neurol 209: 302-312, 2008.
9. McGee AW, Yang Y, Fischer QS, Daw NW and Strittmatter SM: Experience-driven plasticity of visual cortex limited by myelin and Nogo receptor. Science 309: 2222-2226, 2005.

10. Sherman M: Hepatocellular carcinoma: epidemiology, surveillance, and diagnosis. Semin Liver Dis 30: 3-16, 2010.

11. Novak G and Tallerico T: Nogo A, B and C expression in schizophrenia, depression and bipolar frontal cortex, and correlation of Nogo expression with CAA/TATC polymorphism in 3'-UTR. Brain Res 1120: 161-171, 2006.

12. Lie M, Grover M and Whitlon DS: Accelerated neurite growth from spiral ganglion neurons exposed to the Rho kinase inhibitor H-1152. Neuroscience 169: 855-862, 2010.

13. Liu L, Zhang N, Liu J, et al: Lentivirus-mediated siRNA interference targeting SGO-1 inhibits human NSCLC cell growth. Tumour Biol 33: 515-521, 2012

14. Chen YC, Lu DD, Cao XR and Zhang XR: RTN4-C gene expression in hepatocellular carcinoma and its influence on SMMC7721 cell growth and apoptosis. Yi Chuan Xue Bao 32: 891-897, 2005.

15. Kuang E, Wan Q, Li X, Xu H, Zou T and Qi Y: ER stress triggers apoptosis induced by Nogo-B/ASY overexpression. Exp Cell Res 312: 1983-1988, 2006.

16. Zheng H, Xue S, Lian F and Wang YY: A novel promising therapy for vein graft restenosis: overexpressed Nogo-B induces vascular smooth muscle cell apoptosis by activation of the JNK/p38 MAPK signaling pathway. Med Hypotheses 77: 278-281, 2011.

17. Naldini L, Blömer U, Gallay P, et al: In vivo gene delivery and stable transduction of nondividing cells by a lentiviral vector. Science 272: 263-267, 1996.

18. Brummelkamp TR, Bernards R and Agami R: A system for stable expression of short interfering RNAs in mammalian cells. Science 296: 550-553, 2002.

19. Rubinson DA, Dillon CP, Kwiatkowski AV, et al: A lentivirus-based system to functionally silence genes in primary mammalian cells, stem cells and transgenic mice by RNA interference. Nat Genet 33: 401-406, 2003.

20. Sutendra G, Dromparis P, Wright P, et al: The role of Nogo and the mitochondria-endoplasmic reticulum unit in pulmonary hypertension. Sci Transl Med 3: 88ra55, 2011. 